\title{
Additive Manufacturing Under Lunar Gravity and Microgravity
}

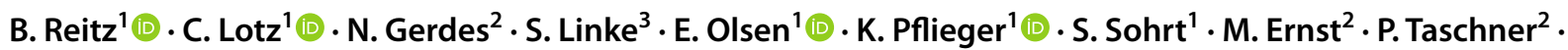 \\ J. Neumann ${ }^{2} \cdot$ E. Stoll ${ }^{3} \cdot$ L. Overmeyer ${ }^{1,2}$ (1)
}

Received: 19 November 2020 / Accepted: 8 March 2021 / Published online: 24 March 2021

(c) The Author(s) 2021

\begin{abstract}
Mankind is setting to colonize space, for which the manufacturing of habitats, tools, spare parts and other infrastructure is required. Commercial manufacturing processes are already well engineered under standard conditions on Earth, which means under Earth's gravity and atmosphere. Based on the literature review, additive manufacturing under lunar and other space gravitational conditions have only been researched to a very limited extent. Especially, additive manufacturing offers many advantages, as it can produce complex structures while saving resources. The materials used do not have to be taken along on the mission, they can even be mined and processed on-site. The Einstein-Elevator offers a unique test environment for experiments under different gravitational conditions. Laser experiments on selectively melting regolith simulant are successfully conducted under lunar gravity and microgravity. The created samples are characterized in terms of their geometry, mass and porosity. These experiments are the first additive manufacturing tests under lunar gravity worldwide.
\end{abstract}

Keywords Additive manufacturing $\cdot$ Lunar gravity and microgravity $\cdot$ Laser melting $\cdot$ Regolith $\cdot$ Einstein-elevator

\section{Introduction}

Additive manufacturing (AM) technologies are well known under Earth's gravity and atmosphere. To pave the way for the colonization of space, those technologies for In-Situ Resource Utilization (ISRU) are an important factor in reducing costs and logistical effort. Additive manufacturing processes have proven to be promising technologies for this purpose in recent years. This paper compares the state of the art of additive manufacturing processes in different environments and presents the feasibility of experiments in the Einstein-Elevator, a next generation drop tower of the Leibniz University Hannover. A first leap for investigation of AM technologies combined with ISRU is melting

B. Reitz

birger.reitz@ita.uni-hannover.de

1 Institut für Transport- und Automatisierungstechnik, Leibniz Universität Hannover, An der Universität 2, Garbsen 30823, Germany

2 Laser Zentrum Hannover e.V, Hollerithallee 8, Hannover 30419, Germany

3 Institut für Raumfahrtsysteme, Technische Universität Braunschweig, Hermann-Blenk-Str. 23, Braunschweig 38108, Germany of lunar regolith simulant by a so-called Mobile Selective Laser Melting (M-SLM) process under lunar and microgravity conditions in the Einstein-Elevator.

After successfully laser melting regolith simulant under vacuum and Earth's gravity, system tests are continued in lunar and microgravity conditions in the Einstein-Elevator. This unique drop tower concept enables substantial progress in research worldwide for future space missions and other fundamental problems. The melted regolith samples are generated under microgravity ( $\mu g \approx 10^{-6} g$ ) - this represents the conditions in orbit or outer space - lunar gravity $(0.16 g)$ and the conditions on Earth $(1 \mathrm{~g})$. The geometric properties of the produced samples are measured. To examine the interior of the samples, they are examined in the computer tomograph (CT) and micrographs are generated. The produced samples have slight differences. In addition, it is shown that the Einstein-Elevator can be used to carry out such experiments successfully and this forms the basis for further experiments in various fields of research.

\section{Additive Manufacturing, Scientific Context}

Additive manufacturing offers many advantages. It is possible to produce new kinds of geometries. Individual parts can be manufactured without great effort, which can be 
carried out on site and produced on demand. In this chapter, the state of research of additive manufacturing techniques under different combinations of environmental conditions (atmosphere/vacuum, Earth's gravity/microgravity) are summarized and evaluated.

\section{Additive Manufacturing Under Gravity and Atmosphere}

Additive manufacturing is a broad area of manufacturing based on layer-wise build-up of different kinds of materials into 3D parts. The parts are designed through computer aided design (CAD), which makes additive manufacturing an ideal tool for rapid prototyping amongst many other applications Guo and Leu (2013). A large number of processes have been established to additively manufacture a wide range of materials. Polymers, ceramics and metals are processed by means of laser or electron beam melting, hot extrusion, polymerisation or binding agents, amongst others. One of the popular methods of AM is Laser Powder Bed Fusion (LPBF), which is already used to process a wide range of materials layerwise in powder form Schmidt et al. (2017). Benefits of LPBF include mechanical properties that are superior to conventionally manufactured parts and high geometrical customizability Frazier (2014); Yap et al. (2015). These advantages make LPBF it greatly applicable in the aerospace and medical industries. The parts manufactured are highly stress resistant and thermally resistant, while being relatively light Guo and Leu (2013); Frazier (2014).

During an LPBF process, there are numerous factors that can affect mechanical properties of created parts Frazier (2014). Physical processes at the microscale, which are hard to control, create different microstructures. Many process parameters such as laser power and scanning speed have to be optimized to obtain desired results Guo and Leu (2013). While significant improvements in this optimization have been already made, there are still research needs to be fulfilled before industries can utilize LPBF at a larger scale under challenging conditions.

\section{Additive Manufacturing Under Gravity and Vacuum}

Currently, manufacturing under vacuum is mainly found in some fields of research as well as in the semiconductor industries and is focused on micro- and nanoscale processes. The field of vacuum application in large scale material processing is only partially investigated for industrial production in the past due to high cost of large scale vacuum systems and missing application scenarios.
Vacuum has a big impact on chemical and physical effects compared to processes under Earth's atmosphere. A fundamental advantage of material processing and additive manufacturing under vacuum is to obtain metal compounds without impurities and the ability to circumvent oxidation on the initial surfaces and resulting microstructures. Thereby, additive manufacturing processes can be combined with promising new materials like magnesium or titanium due to their extremely high oxidation speed in Earth's atmosphere. Without oxidation, the connection of melted layers is improved by a increased wetting behaviour Hagemann et al. (2015). Typically, after the use of chemical flux a complex cleaning process follows. One alternative approach to obtain oxygen-free processing advantages of extreme high vacuum (XHV) is to use process chambers filled with inert gas. It shows excellent results for steel aluminium joints, which indicate that an oxygen-free environment is the key to process optimization Nothdurft et al. (2016). A second approach is the use of silane $\left(\mathrm{SiH}_{4}\right)$ doped inert gas. Hereby, oxygen content in a process can be reduced to XHV equivalent conditions Maier et al. (2020). This approach is comprehensively investigated in the Collaborative Research Center 1368 DFG (2020), which also enables the advantages for additive manufacturing under vacuum conditions to be demonstrated.

The evaporation thresholds are shifted to lower temperatures under vacuum. At the same time, heat dissipation is limited to heat radiation and to conduction into the processed material. This leads to the hypothesis that the required energy for thermally activated processes is reduced in vacuum compared to standard atmospheric conditions. The penetration depth of a weld increases with reduced pressure. Meanwhile, metal vapour generation is reduced and the dynamic behaviour of keyhole and the molten pool differs. Shielding of the laser beam is reduced because the vapour generated by the process dissolves faster into vacuum. Consequently, vacuum enables an improved deep welding process with high aspect ratios due to the increased penetration depths Jiang et al. (2017). These results can be transferred to thermally activated processes in general and give a first idea about the tendency of the following process behaviour.

One purpose of processing regolith by laser melting on the lunar surface is to build up infrastructure with resources on-site. Therefore, fundamental processing characteristics of lunar regolith simulant are investigated in laboratory setups under vacuum and Earth's gravity. Figure 1a shows a mobile experimental setup for a vacuum laser melting experiment. The expected process intensity is affected by reduced heat loss in vacuum and elevated temperature during lunar daylight at approx. $400 \mathrm{~K}$ Fateri and Gebhardt (2015); Gerdes et al. (2019). The In-Situ process result is depictured in Fig. 1b, a succesfully melted sample of regolith simulant. This process is further investigated in this publication under different gravitational conditions for the first time. 


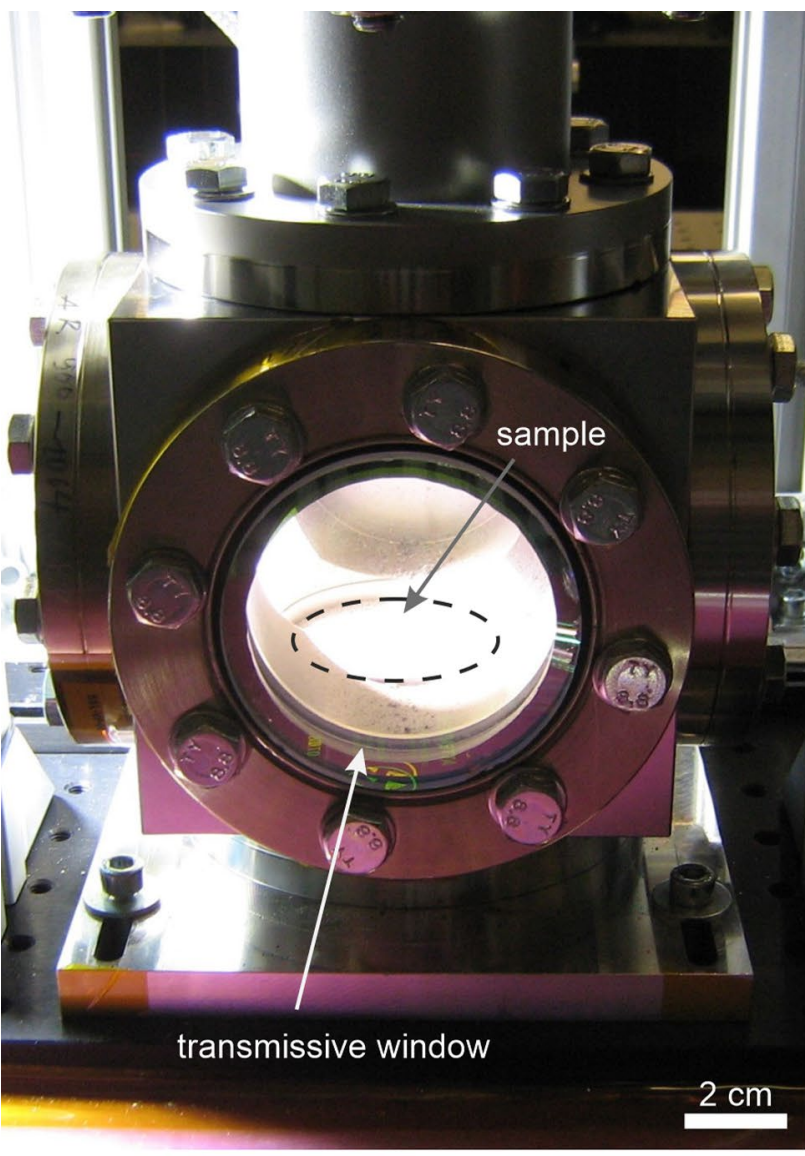

(a)

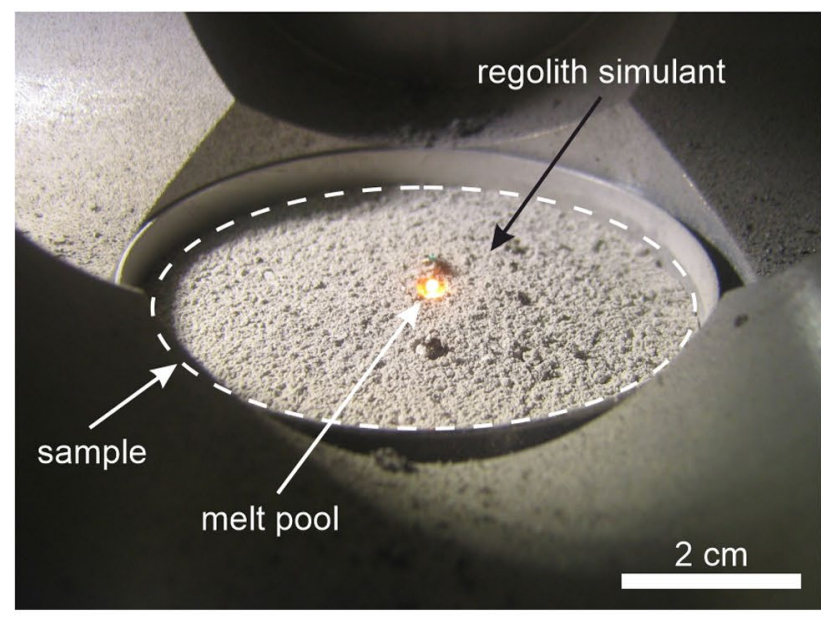

(b)

Fig. 1 (a) Mobile laser melting vacuum chamber stationary installed in laboratory for AM experiments under gravity and vacuum; (b) Regolith simulant powder sample inside the mobile vacuum chamber (Fig. 1a) under gravity with visible meltbath spot

\section{Additive Manufacturing Under Microgravity and Atmosphere}

Modern space exploration is dependent on components processed on Earth, which needs to be transported to space. Exploration of deep space is unrealistic via Earth dependent supply chains, due to long transportation times and high costs. While transportation cost has decreased over recent years, the cost of a Falcon 9 and Dragon capsule mission in 2018 was still $\$ 23.300 / \mathrm{kg}$ Jones (2018). Consequently, a recent research topic is In-Space Manufacturing (ISM). Production in space provides the ability to reduce launch costs, improve crew safety and enable advanced deep space explorations. Traditional manufacturing processes are often not suitable for ISM. They are highly specific in the kind of parts they can produce and are optimized for mass production and almost exclusively subtractive. This is not feasible for the logistics of space exploration. Therefore, it is not costeffective to manufacture metallic components, due to their high density, in space using traditional cutting processes. Additive manufacturing offers a solution to these constraints because it is toolless and very efficient in terms of material consumption Kramer (2002). Made in Space (MIS) installed the first Fused Deposition Modeling printer for commercial use on the International Space Station (ISS) in 2016. They manufactured multiple polymer parts of acrylonitrile butadiene styrene, Polyetherimide/Polycarbonate and High-Density Polyethylene in space Made In Space (2019). In contrast AM of metallic parts in space is not available and thus a promising research topic. NASA is investigating a range of metal manufacturing processes for space applications. To reduce supply flights, parts that are no longer needed must be recycled into new components. It would be conceivable to remelt and reclaim additive components made of plastic or metal in a feedstock recycler.

\section{Additive Manufacturing Under Microgravity and Vacuum}

Since the vacuum is an infinite resource in space, this environment offers completely new opportunities and has many advantages compared to conventional manufacturing processes under Earth's atmosphere. The density in interstellar space is $10^{-20} \mathrm{~kg} / \mathrm{m}^{3}$, which is three orders better than the best laboratory vacuum $\left(10^{-17} \mathrm{~kg} / \mathrm{m}^{3}\right)$ Halliday et al. (2013). An advantage of ISM is that manufactured structures in space does not have to withstand substantial vibrations of the rocket launch. Therefore, structures can be thinner, kilometers-long, and optimized for its primary use without considering stabilisation or packaging Kugler et al. (2017). MIS is developing new additive manufacturing technologies and assembly to create space-structures with an indefinite size on-orbit in the project ArChinaut. 
ARChinaut One is expected to launch in 2022. It is a spacecraft the size of a refrigerator, equipped with a 3D printer and a robotic arm. After being positioned in orbit it will print its own two solar arrays with a length of roughly $10 \mathrm{~m}$. The solar panels can generate up to five times more power than similar-sized spacecrafts Harbaugh and Dunbar (2019). This operation aims to prove the concept of additive manufacturing in space under planetary atmosphere or vacuum. In June 2017, ARCHINAUT demonstrated a successful test in a relevant, space-like thermal vacuum using the $3 \mathrm{D}$ printer of the Archinaut system to extrude a $37.7 \mathrm{~m}$ beam which is many times longer than its manufacturing device Patane et al. (2017). More flexibility in shaping is offered by for example new laser-based AM processes for the production of new components on-site. By selecting suitable laser sources and intensity modulations, it is possible to fully integrate them into vacuum setups, under consideration of thermal management. In space, low power laser sources are already in use for communication and analysis purposes Toyoshima et al. (2015); Seas et al. (2018). The next challenge is the integration of space suitable laser sources for laser-based manufacturing.

\section{Comparison of the Additive Manufacturing Under Different Conditions}

The mentioned four different condition combinations for AM offer their own advantages and disadvantages, as shown in Table 1 . They differ significantly in their degree of knowledge, but also in their component properties such as part size, feature size and process parameters such as speed and material diversity, as well as the technical effort. The summarized state of the art identified a great interest but also the biggest knowledge gap in additive manufacturing under space conditions (atmosphere and vacuum). To fill this knowledge gap, the Einstein-Elevator offers an optimal test environment for experiments under vacuum or atmosphere and adjustable gravity from $\mu-5 g$. The great advantage of the Einstein-Elevator is the easy accessibility and that the costs of tests are much lower than experiments in orbit.

\section{The Experimental Setup}

The Einstein-Elevator at the Hannover Institute of Technology of the Leibniz University Hannover is a next generation drop tower for experiments in adjustable gravity conditions to facilitate more efficient space research on Earth. Today's more complex and highly technological experimental setups require easy accessibility for development work without fully demounting the test chamber. High repetition rates for statistical evaluations to reduce project times to an economical level became possible. For investigating scientific issues, the experimental setup is implemented in a modular experiment carrier. It is $2 \mathrm{~m}$ in height and $1.7 \mathrm{~m}$ in diameter and is constructed with fliexibly adjustable floors and various mounting possibilities for the experimental setup Lotz et al. $(2017,2018,2020)$. The assembled experiment carrier is limited to a maximum of $1,000 \mathrm{~kg}$. Optimization in terms of rigidity and fast oscillation decay is necessary to provide an optimal test environment for the highly sensitive experiments. An optional pressure-tight shell around the experiment carrier serves to separate the electronics of the experimental setup from the vacuum inside the gondola. This setup is fitted into the gondola, a vertically movable vacuum chamber made of carbon fiber reinforced plastic (CFRP). A linear motor accelerates the gondola through a short acceleration phase followed by sudden decoupling and a vertical parabolic trajectory. During the vertical parabolic flight, the gondola travels at a defined distance from the experiment carrier

Table 1 Comparison of additive manufacturing techniques under different operating conditions in general based on the literature review. The $(+)$ stands for a positive valuation, while the (o) and (-) stand for a neutral and a negative valuation respectively

\begin{tabular}{|c|c|c|c|c|}
\hline & \multicolumn{4}{|c|}{ Additive manufacturing under } \\
\hline & atmosphere\& & vacuum\& & atmosphere\& & vacuum\& \\
\hline & gravity & gravity & microgravity & microgravity \\
\hline degree of knowledge & $\begin{array}{l}\text { industrially established } \\
(+)\end{array}$ & $\begin{array}{l}\text { few industrial applications } \\
\text { (o) }\end{array}$ & $\begin{array}{l}\text { state of research } \\
\text { (o) }\end{array}$ & $\begin{array}{l}\text { visionary } \\
(-)\end{array}$ \\
\hline part size & $\begin{array}{l}\text { limited printing volume } \\
(+)\end{array}$ & $\begin{array}{l}\text { vacuum chamber } \\
(-)\end{array}$ & $\begin{array}{l}\text { small printing volume } \\
(-)\end{array}$ & $\begin{array}{l}\text { limited materials } \\
(+)\end{array}$ \\
\hline $\begin{array}{l}\text { feature size } \\
\text { process speed }\end{array}$ & 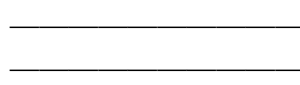 & $\begin{array}{l}\text { ngly dependent on process a } \\
\text { ngly dependent on process a }\end{array}$ & $\begin{array}{l}\text { erial } \\
\text { erial }\end{array}$ & - \\
\hline material diversity & $\begin{array}{l}\text { high variety } \\
(+)\end{array}$ & $\begin{array}{l}\text { non-outgassing materials } \\
\text { (o) }\end{array}$ & $\begin{array}{l}\text { high variety } \\
(+)\end{array}$ & $\begin{array}{l}\text { thermal resistent } \\
(-)\end{array}$ \\
\hline technical effort/costs & $\begin{array}{l}\text { very low } \\
(+)\end{array}$ & $\begin{array}{l}\text { vacuum generation } \\
\text { (o) }\end{array}$ & $\begin{array}{l}\text { high preparation costs } \\
(-)\end{array}$ & \\
\hline
\end{tabular}


such that the carrier is fully decoupled from its environment and is in free fall. The structure of the Einstein-Elevator is illustrated in Fig. 2. A range of technical solutions are utilized to achieve an almost fully automated test procedure and with this, high repetition rates of up to 300 test executions per day in a three-shift operation (one test execution every 4 minutes). This includes automatic centering of the experiment carrier inside the gondola, low volume to be evacuated, permanent data access to the experimental system from the control room as well as an uninterruptible power supply. The precisely controllable linear motor and thus the possibility to conduct experiments not only in microgravity but also in adjustable gravity conditions make the Einstein-Elevator innovative. This means the execution of hypogravity in the range of $\mu-1 g$ (e.g. lunar or Martian gravity), and hypergravity in a range of $1-5 \mathrm{~g}$ are possible to simulate fast acceleration changes during a rocket launch.

To operate, perform and observe experiments inside the gondola, appropriate components are required. Batteries provide uninterrupted power supply as well as adjustable voltage levels and several kilowatts of power during the test execution. They supply the sensor telemetry of the carrier control unit as well as the data communication system and the experiment control unit. Experiments, such as substratefree additive manufacturing require additional systems such as video monitoring and a recording of status data around the experiment like accelerations and rotations Lotz et al. (2018).

For laser melting experiments as described below, a vacuum chamber is designed to accommodate the powder material. Figure 3 shows the setup inside the gondola (left) including the laser housing, high-speed camera and illumination (middle). The use of two chambers along with a quick insertion mechanism allow, for an efficient reloading of the experiment carrier in between flights. Powder material is placed inside a metal vessel (right), that can be inserted into the chamber and is fixed during the experiments using small magnets. Before each experiment, a pressure of $10^{-2} \mathrm{mbar}$ is generated and the powder is compressed with a defined force of $60 \mathrm{~N}$ that is homogeneously applied from above. In the following, the movement of the gondola and the acceleration acting on the experiment are shown using two flights (lunar- $g$ and $\mu g$ ) recorded during the MoONRISE project. The research goal in this project is to bring 3D printing to the moon, using lunar dust as a building material. Figure 4 shows the movement of the gondola during the $0 \mathrm{~g}$ flight. The movement starts at the starting position for parabolic flights. Within the first $0.5 \mathrm{~s}$, the drive accelerates the gondola with $4 \mathrm{~g}$ up to a velocity of $20 \mathrm{~m} / \mathrm{s}$. Including Earth's- $g, 5 g$ are acting on the gondola and the experiment. During a very short phase, the drive creates a distance between the gondola's floor and the experiment carrier feet, while the velocity is already decreasing. This creates a dent in the linear falling

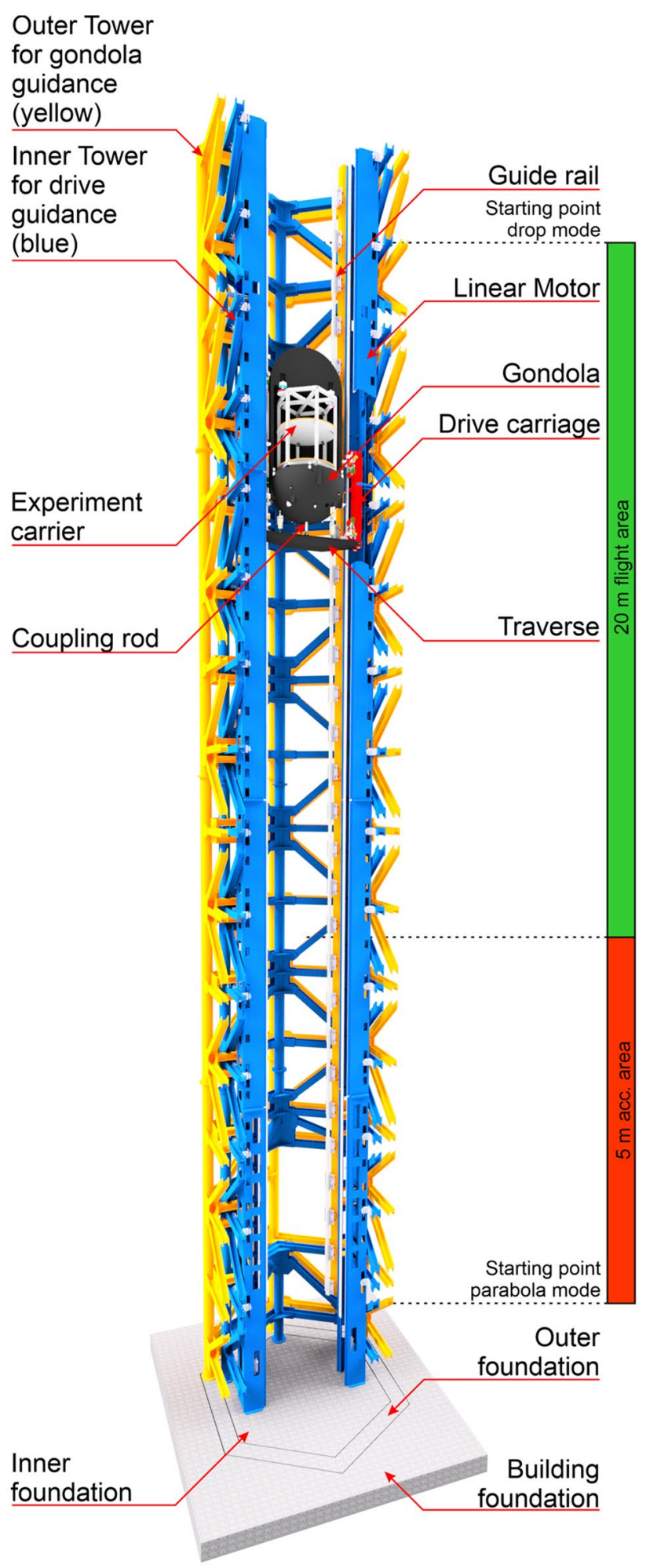

Fig. 2 Design of the Einstein-Elevator

velocity shortly after the maximum speed. After the experiment carrier loses contact to the gondola's floor, it switches from multiple $g$ to $\mu g$. With the initial speed, the gondola 
Fig. 3 Left: Experimental setup inside the Einstein-Elevator's gondola (source: LUH/MarieLuise Kolb), Middle: Flight hardware of the MoonRISE laser system, Right: Melt bead of regolith simulant in the evacuated process chamber

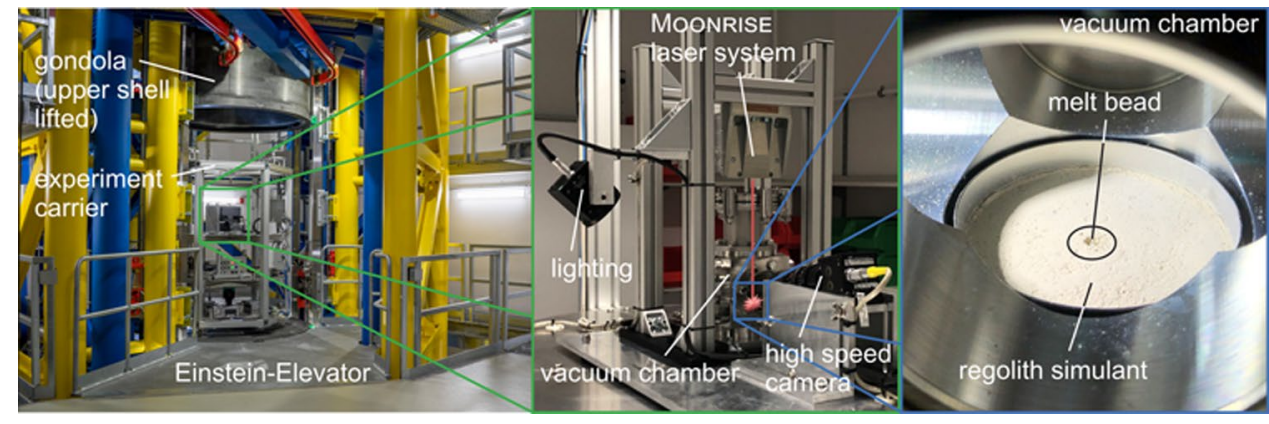

climbs up to $27.4 \mathrm{~m}$ inside the tower. This vertical parabola flight results in $4 \mathrm{~s}$ of free floating for the experiment carrier inside the gondola. Looking closer to the distance between gondola and experiment carrier, after the acceleration phase, the drive creates a distance of $50 \mathrm{~mm}$ within $0.15 \mathrm{~s}$. The free floating boundaries are limited due to the installed dampers. There is no physical connection between a distance of $38 \mathrm{~mm}$ and $68 \mathrm{~mm}$. For the $4 \mathrm{~s}$, the controlled movement of the gondola surrounding the ideally free falling experiment carrier has a maximum deviation of $\pm 2 \mathrm{~mm}$ typically. Shortly before the gondola is braked, the drive decreases the distance between gondola and experiment carrier for a soft approach. The gondola and the experiment carrier on the inside come to a halt at the start position $(0 \mathrm{~m})$ and can be restarted after recentering the carrier, cooling the drives and loading the power storage unit within $4 \mathrm{~min}$.

The general procedure during lunar- $g$ flights are quite similar to the $\mu g$ flights Lotz et al. (2018). The movement starts at the lower starting position. The maximum velocity required to simulate lunar- $g$ is slightly smaller due to the adapted vertical trajectory. At $18.3 \mathrm{~m} / \mathrm{s}$, the drive switches to a slightly accelerated movement, where it pushes the gondola with additional $0.16 \mathrm{~g}$ up to a position of $26.8 \mathrm{~m}$, instead of only compensating air drag and rolling resistance. This increases the experiment time to $4.4 \mathrm{~s}$. After the deceleration at the end of the experiment time, it comes to a halt at the starting position. In contrast to the $\mu g$ flights, the experiment carrier is fixed on the gondola floor the whole time during all flights, simulating different gravitational conditions. For scientific experiments in different gravitational conditions, the acting acceleration forces are important. Figure 4 (small diagram in top right corner) also gives an overview by showing the root mean square (RMS) accelerations $|\mathrm{xyz}|=\vec{g}$ during the previously considered $\mu g$ and the lunar- $g$ flights. As mentioned before, the acceleration at the beginning is slightly smaller for simulating lunar- $g$ with a maximum of $6.9 \mathrm{~g}$ instead of $7.2 \mathrm{~g}$. In addition, the acting time of the acceleration at the beginning is marginally shorter due to the lower initial velocity. The lunar- $g$ experiment lasts a little longer and the deceleration at the end of the flight is slightly reduced. The simulated gravitational conditions become obvious by the almost constant values of $0.16 \mathrm{~g}$ and $\mu$ $g$ between acceleration and deceleration phase. The accelerations are recorded per axis with $10 \mathrm{kHz}$. The signals are filtered to usual $10 \mathrm{~Hz}$ in practice to show the static acceleration forces acting on the experiment without higher vibrational influences and for a better visualization. A further detailed evaluation of the accelerations for a classification of the system, according to McPherson et al. (2017) is not yet possible for these flights. Reasons are that the pre-test carrier has not yet been optimized in terms of vibration and damping behavior and no vacuum in the gondola has been used for acoustic
Fig. 4 Movement of gondola during a $\mu g$ flight (2020-0310UTC08-58-01)

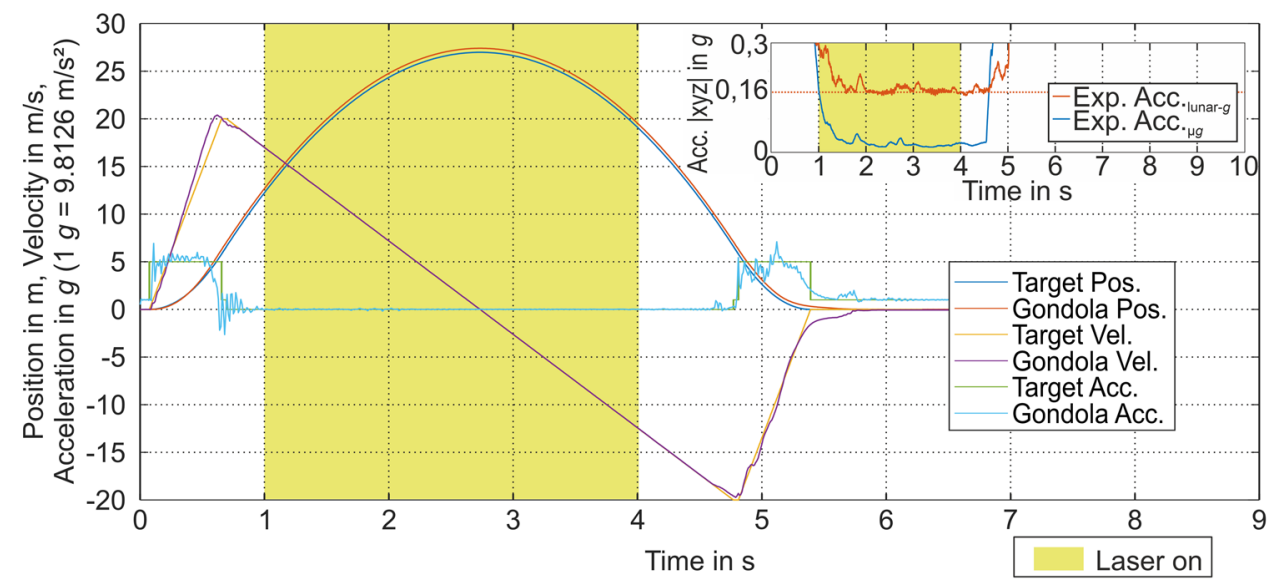


decoupling due to the vacuum incompatible pre-test setup. However, the quality of the $\mu g$ environment and the actual noise level in the Einstein-Elevator will be evaluated in later series of experiments.

\section{Laser Melting of Regolith Simulant Under Lunar and Microgravity Conditions}

Regolith, which can be found everywhere on the surface of the Moon, can be used as building material for a wide range of infrastructures Gerdes et al. (2019); Linke et al. (2020). This mineral material consists of a fine-grained layer with a thickness of several meters and is made of crushed bedrock, volcanic ash and in a small part of the remains of meteorites Jolliff et al. (2006). Regolith has different compositions depending on the region. In the lowland regions, the socalled maria, basalt dominates as the starting material for regolith development. In the highland regions, the so called terrae, anorthosites dominate as bedrock. However, pure forms of regolith, which consist of only one of these components, do not exist due to the mixing processes that have taken place over the course of billions of years. These mixing processes were mainly triggered by meteorite impacts of different sizes Heiken (1991).

For LPBF tests with the MoonRISE laser experiment Linke et al. (2020), a regolith simulant developed and manufactured by TU Braunschweig is used as test material. Original regolith cannot be used due to the small amounts transported from the Moon to earth in the 1960s and 1970s. Regolith simulants are synthetic lunar soils that more or less reproduce the properties of the real lunar soil depending on the application Sibile et al. (2006). Modular system developed at TU Braunschweig enables the processing of regolith simulants which are adapted to specific requirements or regions on the lunar surface. The regolith simulant variant TUBS-I is used for the experiments is described here. TUBS-I combines maria and terrae properties and therefore is a chemical and mineralogical mix of mainly basalt and anorthosite. Such a mixture is very common on the Moon due to the mixing effects described above. Table 2 shows the chemical composition in the form of oxide sums. The average composition of the maria and terrae regoliths found on the Moon serve as a comparison. Local regolith compositions can deviate considerably from this Taylor (1975).

The TUBS-I simulant consists only of lithic particles, i.e. sharp-edged components of crushed rocks. The simulant has a grain size distribution that corresponds to typical lunar regolith. The maximum grain size is $2 \mathrm{~mm}$, but the majority of the particles are significantly smaller, which means that the simulant has an average grain size of $87 \mu \mathrm{m}$ Linke et al. (2020). For the experiments, a bowl with a diameter of $70 \mathrm{~mm}$ is filled with the simulant. The simulant is then solidified with a pressure
Table 2 Average oxidic composition of the maria and terrae regolith Taylor (1975) compared to TUBS-I

\begin{tabular}{lccc}
\hline & Maria in wt\% & TUBS-I in wt\% & Terrae in wt\% \\
\hline $\mathrm{SiO}_{2}$ & 45.4 & 48.7 & 45.5 \\
$\mathrm{TiO}_{2}$ & 3.9 & 1.2 & 0.6 \\
$\mathrm{FeO}$ & 14.1 & 5.6 & 5.9 \\
$\mathrm{MgO}$ & 9.2 & 4.7 & 7.5 \\
$\mathrm{CaO}$ & 11.8 & 11.4 & 15.9 \\
$\mathrm{Na}_{2} \mathrm{O}$ & 0.6 & 3.4 & 0.6 \\
\hline
\end{tabular}

of $0.64 \mathrm{~N} / \mathrm{cm}^{2}$. This solidification ensures that the material remains unchanged in the event of acceleration events in the Einstein-Elevator.

Experiments have already been carried out on melting metals in zero gravity $(0.1 \mathrm{~g})$ during a parabolic flight. Here, the metal powder was held down to a plate by a vacuum pump, the melting experiments were performed in Earth's atmosphere Zocca et al. (2019). Unlike the previous experiment, the aim of the following tests is to prove the functionality of the MoONRISE laser system under different gravity conditions. The punctual regolith melting forms the basis for additive manufacturing with regolith on site. A further implementation of the movement of the laser beam enables the creation of 2D structures, the first step of the LPBF process which was described in Section 2.1. Since the MoonRISE experiment is to be carried out on the surface of the Moon, this ability is very important for the understanding of the regolith processing behavior. In addition to the experiments with lunar gravity of $0.16 \mathrm{~g}$, experiments with $\mu g$ are carried out in the Einstein-Elevator and at $1 g$ in the laboratory. By comparing the samples produced in these three conditions, it is possible to directly identify the effects of the different gravity conditions on the process of sintering and melting regolith. The tests are carried out under all three gravity conditions with an identical setup and laser parameters. Continuous wave irradiation with the laser diode was set for $3 \mathrm{~s}$ at a power of $105 \mathrm{~W}$ and a wavelength of $976 \mathrm{~nm}$. Using an aspherical lens, a spot diameter of approximately $1 \mathrm{~mm}$ was achieved at a working distance of $250 \mathrm{~mm}$.

A high-speed camera was used during the test. This records the melting process at 400 frames per second. It is triggered simultaneously with the start of the test run. The three-second laser process starts half a second after the set gravity is reached. One of these shots can be found in Fig. 5. The melting of the regolith simulant can be seen impressively from the glow of a developing melt bead, the cooling process can also be seen. The glow begins to dim instantaneously when the laser is switched off and goes out completely within $500 \mathrm{~ms}$ of the end of the laser process, and thus before the carrier is touched down in the gondola and the system brakes. A complete solidification of the melt can be assumed, also because the geometry evaluated in the 


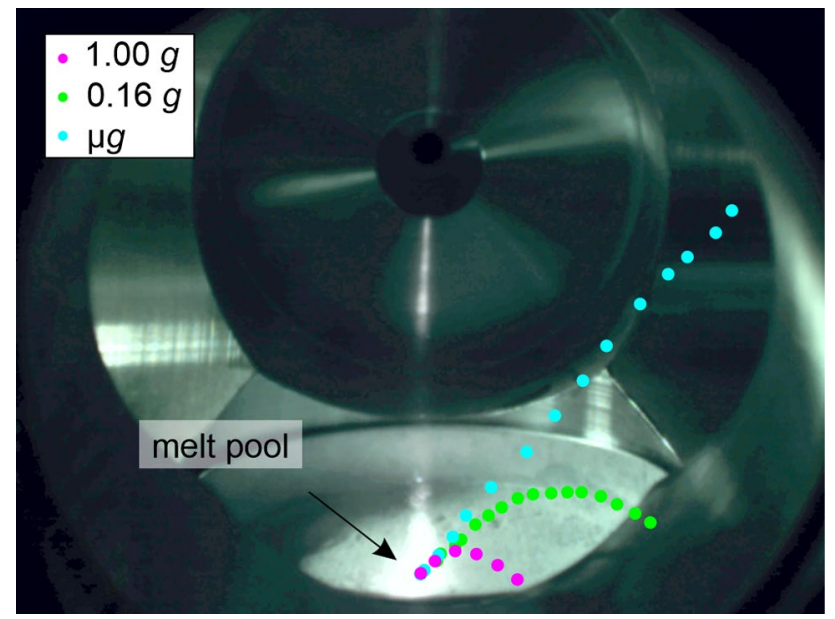

Fig. 5 Trajectories of slow-moving particles detaching from the melt pool

following would otherwise show a preferential orientation of the melt bead deformation.

Differences can be visually observed in the movement of individual melted particles that split off. Depending on the simulated gravitational condition, detached particles fly on differently curved trajectories according to the acceleration acting on them. Figure 5 shows relatively slow particles releasing from the melt pool. Here, attention is paid to a comparable velocity, since the initial gradient of the flight curves depends strongly on the exit velocity.

\section{Geometrical Evaluation}

A total of four samples are evaluated, which are produced at $1 g$, seven samples at $0.16 g$ and six samples at $\mu g$. All samples have a spherical shape and a diameter of approximately $3 \mathrm{~mm}$. The surface is glass-like and regolith particles are baked into the underside of the balls. These particles were not completely melted and are permanently integrated into the sample upon cooling and solidification of the melt. There is no visual difference between all three sample types. A comparison of the geometric properties is shown in Fig. 6a and Fig. $6 \mathrm{~b}$. The figures represent the mean value and the range of the measured values of the evaluated samples. The samples are not ideally round, but resemble an ellipsoid. The mean minimum diameter is $2.74 \mathrm{~mm}$ for the $1 \mathrm{~g}$ samples, $2.86 \mathrm{~mm}$ for the $0.16 \mathrm{~g}$ samples and $2.70 \mathrm{~mm}$ for the $\mu \mathrm{g}$ samples. The mean maximum diameter is $2.99 \mathrm{~mm}$ for the $1 \mathrm{~g}$ samples, $3.19 \mathrm{~mm}$ for the $0.16 \mathrm{~g}$ samples and $3.24 \mathrm{~mm}$ for the $\mu g$ samples. The values for all three sample types are therefore close to each other. Higher scatter of the $\mu g$ samples compared to the other two sample types is to be mentioned. The roundness of the samples is calculated from the ratio of maximum to minimum diameter as follows:
$R_{e}=\frac{D_{\max }-D_{\min }}{2}$

There is no preferred direction for the maximum and minimum diameters. The surface structure shows which side of the sample is at the top and thus facing the laser, since it has a smooth, shiny surface. The underside of the sample contains embedded unmelted particles and therefore appears rough and dull. Therefore, a clear assignment of the position of the sample is possible even after removal from the powder bed. The higher scattering of the $\mu g$ samples is also evident when considering the roundness of the samples, see Fig. 6c. While the samples produced at $1 \mathrm{~g}$ and $0.16 \mathrm{~g}$ have an average roundness of 0.126 and 0.133 with a comparatively low scatter, the mean value of $\mu g$ with 0.268 is twice as high and the scatter is significantly larger. Basically, rounder samples would be expected at $\mu g$. The reason for this effect is unknown and must be investigated in future experiments. One possible explanation is the short duration of the experiments, so that the spheres cooled and solidified before they could assume an ideally round shape. Another cause could be the movement of the bubbles within the melt during the solidification process, which led to distortion effects.

In terms of mass and volume, see Fig. 6d and Fig. 6e, all sample types show comparable values. The mean values for the mass are $17.30 \cdot 10^{-3} \mathrm{~g}$ for the $1 \mathrm{~g}$ samples, $21.29 \cdot 10^{-3} \mathrm{~g}$ for the $0.16 \mathrm{~g}$ samples and $17.63 \cdot 10^{-3} \mathrm{~g}$ for the $0 \mathrm{~g}$ samples. The volume is $12.21 \mathrm{~mm}^{3}$ for the $1 \mathrm{~g}$ samples, $14.68 \mathrm{~mm}^{3}$ for the $0.16 \mathrm{~g}$ samples and $13.47 \mathrm{~mm}^{3}$ for the $0 \mathrm{~g}$ samples. It is again to be mentioned that the largest scatter is measured in the samples produced under $\mu g$.

The density $\rho_{S}$ can be calculated from the measured mass and the volume of the samples, which, using the known solid density $\rho_{R}$ of the regolith simulant of $2.84 \mathrm{~g} / \mathrm{cm}^{3}$, allows the porosity $\Phi$ to be calculated using the following equation:

$\Phi=\left(1-\frac{\rho_{S}}{\rho_{R}}\right) \cdot 100 \%$

The porosity gives an indication about the internal structure and the proportion of voids in the samples. For all samples, a high proportion of voids is found, which make up about half of the measured volume, see Fig. 6f. The porosity is $49.70 \%$ for the samples produced with $1 \mathrm{~g}$ and $46.77 \%$ for the samples produced with $0.16 g$ and at $\mu g$ it is $53.93 \%$. The calculated porosities coincide with the observations from the micrographs, in which bubbles of different sizes are found, which are embedded in a glass-like matrix. The cause of the formation of the bubbles can be outgassing of volatile components from the regolith simulant or residual air which is contained in the cavities between the particles. The cooling and solidification of the melts takes place so quickly that the bubbles can not emerge on the surface. 
Fig. 6 Geometrical evaluation of the produced samples

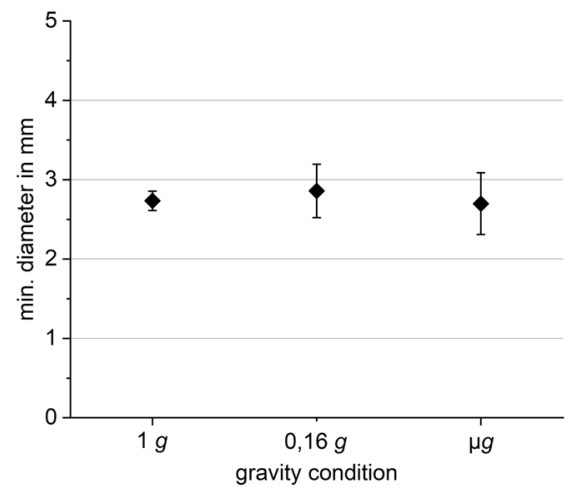

(a) Minimum diameter of the samples

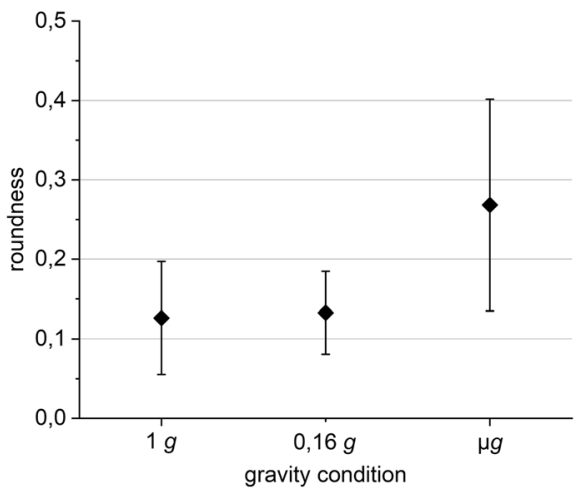

(c) Roundness of the samples according to equation 1

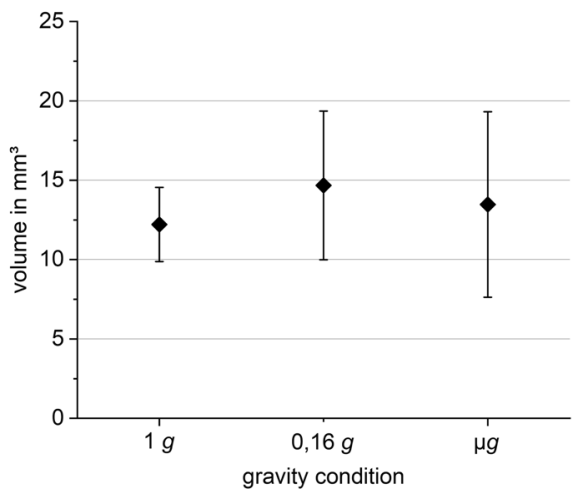

(e) Evaluation of the volume of the samples

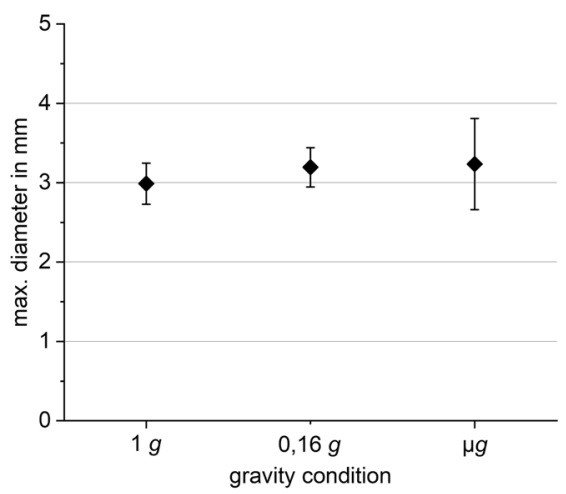

(b) Maximum diameter of the samples

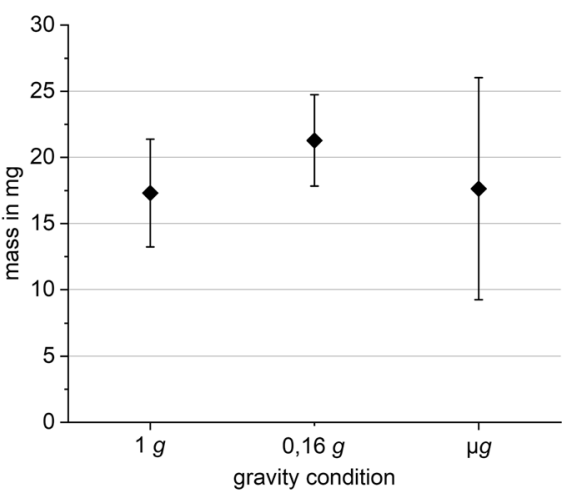

(d) Evaluation of the mass of the samples

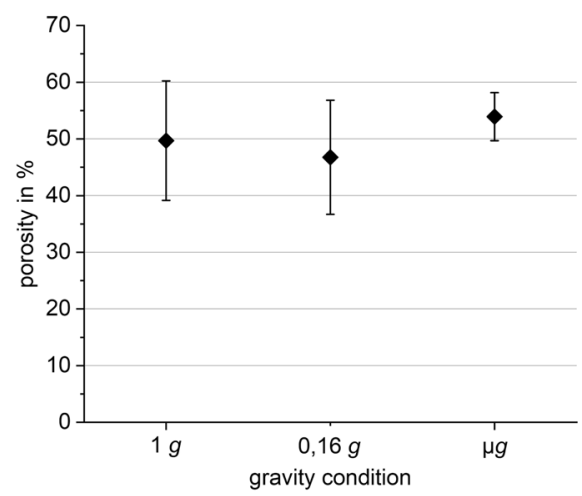

(f) Calculated porosity of the samples

\section{Microsections and Computer Tomography Results}

A microsection and a thin section of a sample produced at $\mu g$ can be seen in Fig. 7. Some of the typical bubbles that are embedded in a glass matrix are visible. The green coloured glass itself is homogeneous and contains no further unmelted particles. The samples produced at $1 \mathrm{~g}$ and $0.16 \mathrm{~g}$ also have an identical internal structure, so that the image is representative for these too.
Figure 8a, Fig. 8b and Fig. 8c show images of CT-scans from three samples that were each processed under different gravitational conditions. The produced samples are round and show some irregularities on the surface. Each of the samples contains pores that are open to the outside. The cross-sectional images further reveal a strong variation in pore size. Many of the pores are interconnected.

While these images give insight into the pore size and distribution, they cannot be used to infer a statistically 

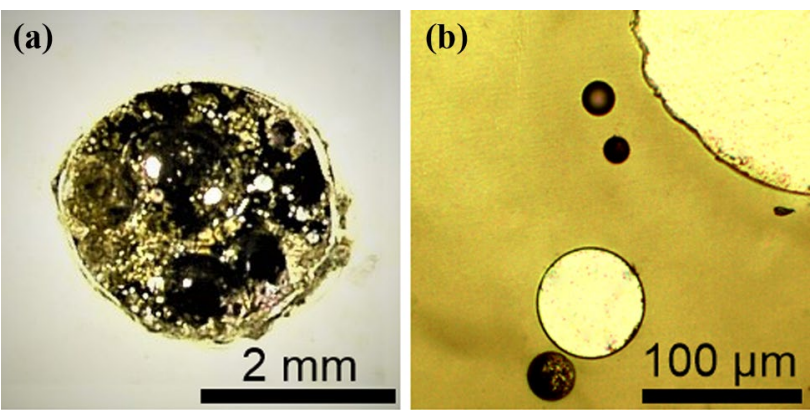

Fig. 7 (a) Microsection of a sample produced in $\mu g$; (b) thin section of the same sample

significant difference between samples produced under different gravitational conditions. There is a large inherent spread during the processing of the samples and the eight CT-scans (four at $1 g$, two at each $0.16 g$ and $\mu g$ ) are not sufficient to unveil significant differences.

\section{Further Investigations}

The tests showed that the LPBF method used in the MoonRISE experiment works reliably under different gravity conditions and delivers comparable results. This provides important evidence that the experiment can be successfully performed on the lunar surface. However, the small number of samples in this first series of experiments does not allow a more in-depth investigation of the processes. It does not allow a statistically relevant evaluation. Therefore, the tests described are to be assessed as a successful proof of concept. The next step is to carry out a more extensive test program, in which the experience of the first test series must be incorporated. For example, the influence of drying the regolith simulant to avoid outgassing during the experiment is to be examined in order to understand the influence of volatile constituents on the formation of voids. For the tests described, the simulant is dried in advance at $973 \mathrm{~K}$, but it is possible that residues of water are present in the mineral crystals of the simulant particles. Furthermore, the experiments are to be designed in a way that the material properties of the resulting material can also be determined from the samples. Since the Moonrise process is designed for the construction of habitats and other infrastructures on the Moon, the material properties are very important to allow suitable designs. Additionally, the influence of other regolith compositions and of other particle types, such as the thermally altered components (glasses, agglutinates), has to be examined. Such thermally altered particles can make up portions of $>50 \%$ in the regolith. These types of particles are very likely to affect the properties of the samples and the material.

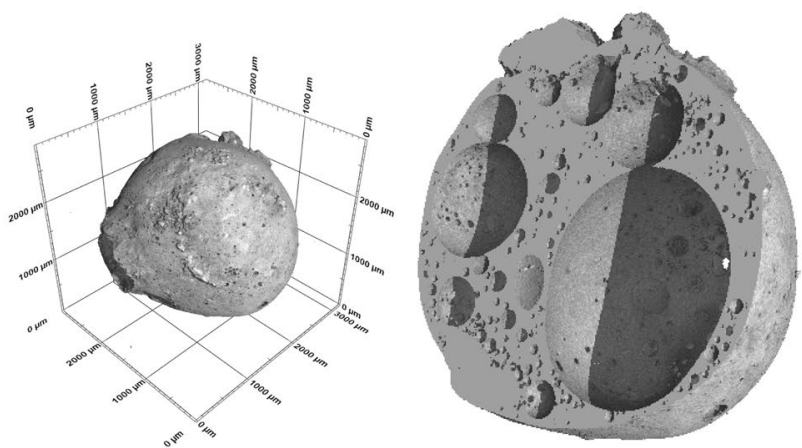

(a) Samples processed at $1 \mathrm{~g}$
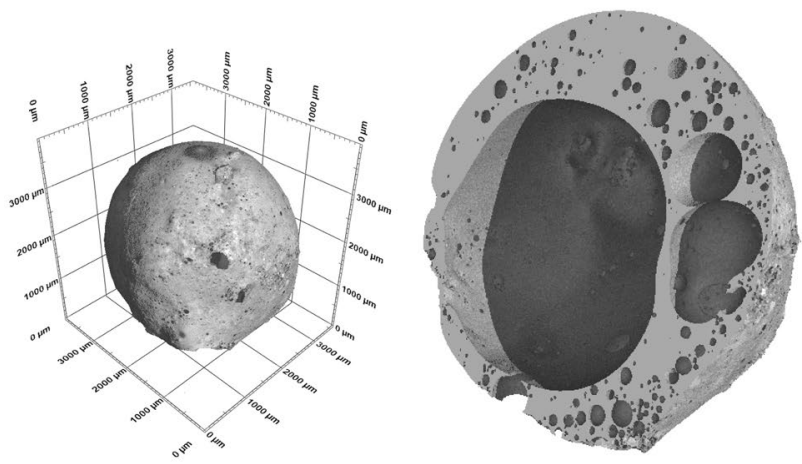

(b) Samples processed at $0.16 \mathrm{~g}$
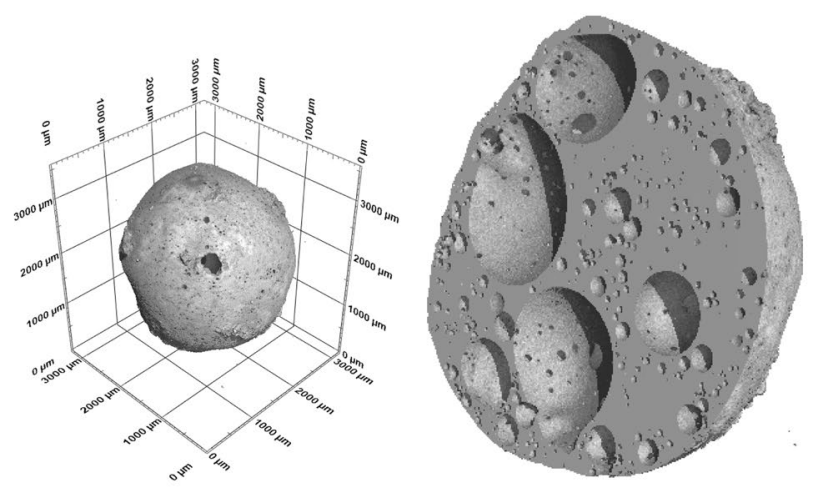

(c) Samples processed at $\mu \mathrm{g}$

Fig. 8 Images from CT-scans of samples processed at various gravity conditions

\section{Conclusion}

The state of the art of additive manufacturing under different environmental conditions is presented and the resulting need for experiments under varying gravitational and atmospheric conditions is demonstrated. The EinsteinElevator offers the novel ability to carry out experiments under adjustable gravitational conditions $(\mu-5 g)$ as well as the necessary infrastructure and accesibility to adjust these conditions at a low cost. Experiments can be integrated into 
the Einstein-Elevator and the flight curve of the experiments show that the required conditions were achieved. The world's first additive manufacturing process under lunar conditions is carried out and presented. The melting experiments of regolith simulant under different gravitational conditions do not show any major differences among the samples regarding geometry, mass and porosity. In the future, a vibration-optimized carrier will be used on the system side and a vacuum will be applied for acoustic decoupling to reduce the noise level. On the experimental side, temperatures and pressures (partial pressure probably increases during melting) during the melting process are to be recorded and analysed. However, it is shown that the hardware for the results of this test series is promising and form the basis for further investigations.

Acknowledgements Open Access funding enabled and organized by Projekt DEAL. This research was funded by the Deutsche Forschungsgemeinschaft (DFG) under Germany's Excellence Strategy within the Cluster of Excellence PhoenixD (EXC 2122, Project ID 390833453). The authors would also like to thank the DFG and the Lower Saxony state government for their financial support for building the Hannover Institute of Technology (HITec) and the Einstein-Elevator (NI1450004, INST 187/624-1 FUGB). The research project MoonRISE is funded by the VolkswagenStiftung (Az. 94647 and Az. 94890).

Data Availability Statement The dataset of samples analyzed during the current study are available from the corresponding authors on reasonable request.

\section{Declarations}

Conflicts of interest The authors declare that they have no conflict of interest.

Open Access This article is licensed under a Creative Commons Attribution 4.0 International License, which permits use, sharing, adaptation, distribution and reproduction in any medium or format, as long as you give appropriate credit to the original author(s) and the source, provide a link to the Creative Commons licence, and indicate if changes were made. The images or other third party material in this article are included in the article's Creative Commons licence, unless indicated otherwise in a credit line to the material. If material is not included in the article's Creative Commons licence and your intended use is not permitted by statutory regulation or exceeds the permitted use, you will need to obtain permission directly from the copyright holder. To view a copy of this licence, visit http://creativecommons.org/licenses/by/4.0/.

\section{References}

DFG Deutsche Forschungsgesellschaft, SFB 1368: Oxygen-free production processes and local mechanisms in oxygen-free atmosphere for the development of sustainable production techniques and manufacturing processes, (2020) https://gepris.dfg.de/gepris/projekt/394563137?1 anguage $=\mathrm{en}$
Fateri, M., Gebhardt, A.: Process parameters development of selective laser melting of lunar regolith for on-site manufacturing applications. Int. J. Appl. Ceram. Technol. (2015) https://doi.org/10.1111/ijac.12326

Frazier, W.E.: Metal additive manufacturing: A review. J. Mater. Eng. Perform. (2014) https://doi.org/10.1007/s11665-014-0958-z

Gerdes, N., Fokken, LG., Linke, S., Kaierle, S., Suttmann, O., Hermsdorf, J., Stoll, E., Trentlage, C., Overmeyer, L.: Selective laser melting for processing of regolith in support of a lunar base. J. Laser Appl. (2019) https://doi.org/10.2351/1.5062614

Guo, N., Leu, M.C.: Additive manufacturing: technology, applications and research needs. Front. Mech. Eng. (2013) https://doi.org/10. 1007/s11465-013-0248-8

Hagemann, R., Rust, W., Noelke, C., Kaierle, S., Overmeyer, L., Rau, T., Huegl, S., Wesling, V., Wolkers, W.: Möglichkeiten der funktionellen lokalen Konfiguration von Mikroaktoren aus Nickel-Titan für medizinische Implantate durch selektives Laserstrahlmikroschmelzen, Neue Entwicklungen in der Additiven Fertigung, Springer Berlin Heidelberg, pp 109-124 (2015)

Halliday, D., Resnick, R., Walker, J.: Fundamentals of physics extend, 10th edn. Wiley, New York (2013)

Harbaugh, J., Dunbar, B.: Archinaut one: National aeronautics and space administration, (2019) https://www.nasa.gov/mission_pages/tdm/ archinaut/index.html

Heiken, G.H.: Lunar sourcebook: A user's guide to the moon. Univ. Press, Cambridge (1991)

Jiang, M., Tao, W., Chen, Y.: Laser welding under vacuum: A review. Appl. Sci. (2017) https://doi.org/10.3390/app7090909

Jolliff, B.L., Wieczorek, M.A., Shearer, C.K., Neal, C.R.: New views of the moon, Reviews in mineralogy and geochemistry, vol. 60 . Mineralogical Society of America, Chantilly, Va (2006)

Jones, H.W.: The recent large reduction in space launch cost, 48th International Conference on Environmental Systems, NASA Ames Research Center (2018)

Kramer, H.J.: Iss utilization: Amf (additive manufacturing facility), (2002) https://directory.eoportal.org/web/eoportal/satellite-missions/i/iss-amf

Kugler, J., Cherston, J., Joyce, E.R., Shestople, P., Snyder, M.P.: Applications for the archinaut in space manufacturing and assembly capability. In: AIAA SPACE and Astronautics Forum and Exposition, American Institute of Aeronautics and Astronautics (2017) https://doi.org/10.2514/6.2017-53652017-5365

Linke, S., Windisch, L., Kueter, N., Wanvik, J.E., Voss, A., Stoll, E., Schilde, C., Kwade, A.: Tubs-m and tubs-t based modular regolith simulant system for the support of lunar isru activities. Planet. Space Sci. (2020) https://gepris.dfg.de/gepris/projekt/ 394563137? language $=$ en

Lotz, C., Froböse, T., Wanner, A., Overmeyer, L., Ertmer, W.: Einstein-elevator: A new facility for research from $\mu \mathrm{g}$ to $5 \mathrm{~g}$. Gravit. Space Res. 5(2), 11-27 (2017)

Lotz, C., Wessarges, Y., Hermsdorf, J., Ertmer, W., Overmeyer, L.: Novel active driven drop tower facility for microgravity experiments investigating production technologies on the example of substrate-free additive manufacturing. Adv. Space Res. (2018) https://gepris.dfg. de/gepris/projekt/394563137?language $=$ en

Lotz, C., Gerdesm, N., Sperling, R., Lazar, S., Linke, S., Neumann, J., Stoll, E., Ertmer, W., Overmeyer, L.: Tests of additive manufacturing and other processes under space gravity conditions in the Einstein-Elevator. Logistics Journal: Proceedings, Vol. 2020, (2020) https://gepris.dfg.de/gepris/projekt/394563137?language=en

Made In Space Additive manufacturing facility: $3 \mathrm{~d}$ printing the future in space, (2019) https://gepris.dfg.de/gepris/projekt/ 394563137? language $=$ en

Maier, H.J., Herbst, S., Denkena, B., Dittrich, M.A., Schaper, F., Worpenberg, S., Gustus, R., Maus-Friedrichs, W.: Towards dry machining of titanium-based alloys: A new approach using an 
oxygen-free environment, Metals 10(9):1161, (2020) https:// gepris.dfg.de/gepris/projekt/394563137?language =en

McPherson, K., Kelly, E., Keller, J., Ibrahim, A., Wagner, E., Hrovat, K.: Analysis of Vibratory Data Collected by the Space Acceleration Measurement System (SAMS) on Blue Origin. Gravit. Space Res. 5(2), (2017)

Nothdurft, S., Springer, A., Kaierle, S., Ross, J., Stonis, M.: Laser soldering and brazing of steel-aluminum sheets for tailored hybrid tubes. J. Laser Appl. (2016) https://gepris.dfg.de/gepris/projekt/ 394563137? language $=\mathrm{en}$

Patane, S., Joyce, E.R., Snyder, M.P., Shestople, P.: Archinaut: In-space manufacturing and assembly for next-generation space habitats, AIAA SPACE and Astronautics Forum and Exposition, American Institute of Aeronautics and Astronautics, Reston, Virginia, p 2016, (2017) https://gepris.dfg.de/gepris/projekt/394563137?language=en

Schmidt, M., Merklein, M., Bourell, D., Dimitrov, D., Hausotte, T., Wegener, K., Overmeyer, L., Vollertsen, F., Levy, G.N.: Laser based additive manufacturing in industry and academia, CIRP Annals. (2017) https://gepris.dfg.de/gepris/projekt/394563137?language=en

Seas, A., Robinson, B., Shih, T., Khatri, F., Brumfield, M.: Optical communications systems for nasa's human space flight missions, International Conference on Space Optics - ICSO 2018, 2018, Chania, Greece, vol ICSO 2018, National Aeronautics and Space Administration (2018) https://gepris.dfg.de/gepris/projekt/394563137? language $=\mathrm{en}$

Sibille, L., Carpenter, P., Schlagheck, R., French, R.A.: Lunar Regolith Simulant Materials: Recommendations for Standardization, International Conference on Space Optics (2006)
Taylor, S.R.: Lunar Science: Scientific Results and Insights from the Lunar Samples. Elsevier Science, Burlington (1975)

Toyoshima, M., Fuse, T., Kolev, D.R., Takenaka, H., Munemasa, Y., Iwakiri, N., Suzuki, K., Koyama, Y., Kubooka, T., Akioka, M., Kunimori, H.: Current status of research and development on space laser communications technologies and future plans in nict, 2015 IEEE International Conference on Space Optical Systems and Applications (ICSOS), Piscataway, NJ, (2015) https://doi.org/ 10.1111/ijac. 12326

Yap, C.Y., Chua, C.K., Dong, Z.L., Liu, Z.H., Zhang, D.Q., Loh, L.E., Sing, S.L.: Review of selective laser melting: Materials and applications. App. Phys. Rev. (2015) https://doi.org/10.1111/ijac.12326

Zocca, A., Lüchtenborg, J., Mühler, T., Wilbig, J., Mohr, G., Villatte, T., Léonard, F., Nolze, G., Sparenberg, M., Melcher, J., Hilgenberg, K., Günster, J.: Enabling the 3D Printing of Metal Components in $\mu$-Gravity. Adv. Mater. Technol. 4(10), (2019) https://doi. org/10.1111/ijac.12326

Publisher's Note Springer Nature remains neutral with regard to jurisdictional claims in published maps and institutional affiliations. 